\title{
A Field-Portable Membrane Introduction Mass Spectrometer for Real-time Quantitation and Spatial Mapping of Atmospheric and Aqueous Contaminants
}

\author{
Ryan J. Bell, ${ }^{1}$ Nicholas G. Davey, ${ }^{1,2}$ Morten Martinsen, ${ }^{1,3}$ Christian Collin-Hansen, ${ }^{4}$ \\ Erik T. Krogh, ${ }^{1,2}$ Christopher G. Gill ${ }^{1,2}$ \\ ${ }^{1}$ Applied Environmental Research Laboratories (AERL), Chemistry Department, Vancouver Island University, Nanaimo, BC, Canada \\ ${ }^{2}$ Chemistry Department, University of Victoria, Victoria, BC, Canada \\ ${ }^{3}$ Chemistry Department, Norwegian University of Science and Technology (NTNU), Trondheim, Norway \\ ${ }^{4}$ Statoil Petroleum ASA, TPD RDI Frontier Developments, Trondheim, Norway
}

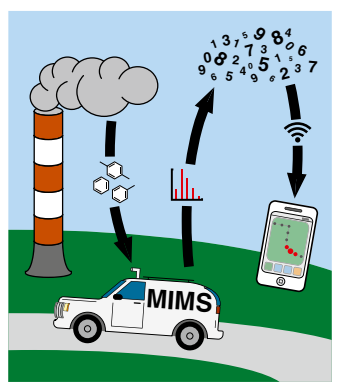

Abstract. Environmental concentrations of volatile and semivolatile organic compounds (VOC/SVOCs) can vary dramatically in time and space under the influence of environmental conditions. In an industrial setting, multiple point and diffuse sources can contribute to fugitive emissions. Assessments and monitoring programs using periodic grab sampling provide limited information, often with delay times of days or weeks. We report the development and use of a novel, portable membrane introduction mass spectrometry (MIMS) system capable of resolving and quantifying VOC and SVOCs with high spatial and temporal resolution, in the field, in real-time. An electron impact ionization cylindrical ion trap mass spectrometer modified with a capillary hollow fiber polydimethylsiloxane membrane interface was used for continuous air and water sampling. Tandem mass spectrometry and selected ion monitoring scans performed in series allowed for the quantitation of target analytes, and full scan mode was used to survey for unexpected analytes. Predeployment and in-field external calibrations were combined with a continuously infused internal standard to enable real-time quantitation and monitor instrument performance. The system was operated in a moving vehicle with internet-linked data processing and storage. Software development to integrate MIMS and relevant metadata for visualization and geospatial presentation in Google Earth is presented. Continuous quantitation enables the capture of transient events that may be missed or under-represented by traditional grab sampling strategies. Real-time geospatial maps of chemical concentration enable adaptive sampling and in-field decision support. Sample datasets presented in this work were collected in Northern Alberta in 2010-2012.

Key words: VOC/SVOC, Membrane introduction mass spectrometry (MIMS), Emissions, Alberta oil sands, Mobile mass spectrometry, Real time quantitative mapping

Received: 15 August 2014/Revised: 9 October 2014/Accepted: 11 October 2014/Published Online: 5 December 2014

\section{Introduction}

$\mathrm{M}$ any processes, both natural and anthropogenic, are associated with the release of volatile and semivolatile organic compounds (VOC/SVOCs) to the environment [1]. When considering hydrocarbon extraction and processing, these emissions may be atmospheric [2] or aqueous [3], can be from point or diffuse sources [4], and are potentially harmful to

Correspondence to: Erik T. Krogh; e-mail: Erik.Krogh@viu.ca, Christopher G. Gill; e-mail: Chris.Gill@viu.ca nearby residents, biota, and workers [5]. Additionally, contaminant concentrations in the environment can be very dynamic as plumes migrate, especially under the influence of mixing events driven by meteorological and hydrological processes $[6,7]$. Grab sample collection (e.g., bottles, canisters, and sorbent traps) are often the primary sampling method used for current environmental monitoring programs and, although quantitative in nature, measured concentrations reflect a single point in space and time (or integrated over a specific time window), predominantly in an 'off-line' manner. The time 
and expense required by these strategies often results in monitoring programs with relatively low data density that are limited in scope, and that can fail to provide information for making timely corrective decisions. Furthermore, improper documentation of baseline concentrations to compare industrially affected areas can lead to controversial situations that are difficult to assess [8, 9]. As a result, grab sample collection programs are often very limited and frequently serve only to meet current regulatory compliance requirements.

The advent of portable chemical sensing technology has enabled a new framework for environmental monitoring, where it is possible to take high precision, high accuracy chemical measurements to the field [10-18]. Many of these analytical techniques, such as membrane introduction mass spectrometry (MIMS), can provide continuous monitoring time series data that resolve multiple analyte concentrations simultaneously [16, 17, 19-24]. When operated on a mobile platform, these instruments can track transient concentration excursions in both spatial and temporal domains that may be missed by grab sampling strategies [24]. Continuous datasets allow the analyst to better understand the spatial boundaries of contaminant plumes as well as temporal changes due to dilution, mixing, and/or processing. For example, directly comparing plume intensities and background intensities in a single dataset can reduce the possibility of obtaining both false negative and/or positive measurements made in dynamic environments. Furthermore, with this information collected in realtime, sampling decisions and corrective actions can be made in a timely manner, helping to mitigate liability for both resource management and environmental stakeholders.

Our research has involved the development of a portable MIMS system for the continuous analysis of VOCs and SVOCs in both atmospheric and aqueous samples that greatly improves upon our early work in this area $[16,17,20]$. In general, MIMS systems allow continuous sampling of VOC/SVOC analytes as they diffuse through a semiselective membrane, whereupon simultaneous quantification of multiple copermeating molecules occurs by mass spectrometry [20, 25, 26]. The use of hydrophobic membranes, such as polydimethylsiloxane (PDMS), impedes the diffusion of ionic and hydrophilic compounds, while pre-concentrating hydrophobic compounds, enhancing their permeation. Thus, MIMS systems employing PDMS membranes yield sensitive and selective determinations of VOC/SVOCs in both aqueous and gaseous samples. Because the membrane allows analytes to pass simultaneously as a mixture, it is important for quantitative analyses that the mass spectrometer can resolve each analyte without interference. Analyte interferences can be reduced through the use of tandem mass spectrometric (MS/MS) techniques. In addition, corrections for known interferences can be applied by using simultaneous measurements for these interfering compounds and through inspection of analyte response times [27].

The collection of continuous real-time datasets affords additional challenges and opportunities for data storage, presentation, and interpretation. In-field data collection is eased through the use of cellular networks and cloud-based storage. These systems allow any user with an internet connection to inspect the data at any time, while simultaneously creating an integrated data backup system. Data presentation and interpretation is eased through the use of free Geographic Information System (GIS) applications, such as Google Earth. Using mass spectrometric data that is both time- and location-stamped, we are able to provide real-time chemical concentrations that are geospatially mapped. In addition to providing environmental protection through rapid screening and continuous monitoring applications, this approach can be used to inform intelligent adaptive sampling strategies, delineate plumes or contaminated sites, identify point sources, quantify fugitive emissions, monitor process efficiencies, and provide discrete molecular information about complex mixtures (e.g., chemical fingerprinting).

In summary, we present the development and application of a field portable, battery operated MIMS system based upon a quadrupole ion trap that utilizes MS/MS for direct, on-line, low level (e.g., $\mathrm{ppb}_{\mathrm{v}} / \mathrm{ppb}$ ) analyte measurements in air and water samples. This flexible measurement system is compact and can be operated in an automobile for mobile measurements. Since volatile and semivolatile analytes pervaporate through the membrane interface from a sample as a mixture, the full resolution of isobaric isomers may not be possible, and other techniques involving chromatographic separations may be more appropriate. Additionally, higher sensitivity for analytes in gaseous samples can be achieved via methods such as proton transfer reaction mass spectrometry (PRTMS) $[18,28]$ and selected ion flow tube mass spectrometry (SIFT-MS) [29, 30]. Despite these potential drawbacks, we have successfully employed mobile MIMS to provide continuous geospatially resolved quantitative information for target analytes in both air and water, using in-house developed data management software to process and visualize the data in real-time using cellular network communications.

\section{Methods and Materials}

\section{Overview}

A commercially available, portable cylindrical ion trap mass spectrometer system (Model Griffin 400; FLIR, West Lafayette, IN, USA), was modified in-house for in-field measurements by the addition of a MIMS sampling interface and internal standard (IS) infusion system, similar to that described previously [16]. In addition to the MIMS sampling system (which is described in detail below), the system also included several peripheral monitoring devices. A global positioning system (GPS, QStarz, Model BT-Q1000XT; Taipei, Taiwan) was used for geo-location and accurate time-stamping of all collected data. A cellular network card installed in the data system computer (Model M6600; Dell, Round Rock, TX, USA) was used to provide cloud data storage (Dropbox, San Francisco, CA, USA) and continuous data backup in the field. A portable weather meter (Kestrel, Model 4500; Birmingham, MI, USA) was used periodically to determine meteorological data, including wind speed, wind direction, humidity, temperature, and barometric pressure. Although not utilized for the 
presented study, a video camera (Model HERO2; GoPro Inc., San Mateo, CA, USA) was incorporated to allow the potential creation of a video log of the surrounding environment during data collection events, to allow retrospective assessment of the data collection environment.

\section{Membrane Introduction Mass Spectrometer}

The cylindrical ion trap utilizing electron impact ionization (EI) was selected for use as the mass analyzer because of its compact, ruggedized, and field-portable design, and its ability to perform MS/MS for increased selectivity. Furthermore, the system is capable of multiple types of mass scan techniques in an interlaced series, allowing cycled quantitative analyte scans to be performed in sequence with general survey (full) scans. The entire mass spectrometer was encased in approximately $1.6 \mathrm{~m}^{2}$ of $0.1 \mathrm{~mm}$ thick nickel alloy foil, or mu-metal $(\mu$ $\approx 50,000$ ) (product number $8912 \mathrm{~K} 32$; McMaster Carr, Elmherst, IL, USA) to reduce the effects of the earth's magnetic field upon instrumental sensitivity. For additional information regarding the influence of magnetic fields upon portable EI mass spectrometers, the reader is directed to an article specifically addressing the topic, also in this issue of JASMS. The instrument was equipped with a temperature-controlled MIMS interface that replaced the supplied gas chromatograph system. For this work, the MIMS interface was constructed using a PDMS hollow fiber membrane $(0.94 \mathrm{~mm}$ o.d., $0.51 \mathrm{~mm}$ i.d., $10 \mathrm{~cm}$ length, Silastic brand; Dow Corning, Midland, MI, USA) and a flow-over sample cell design. A similar experimental arrangement has been described previously [16], though a major difference in design is the use of an in-house constructed aluminum block heater to maintain a constant membrane temperature. The lumen (permeate side) of the membrane was swept with a helium carrier gas $(\sim 1 \mathrm{~mL} / \mathrm{min})$, provided by a miniature $16 \mathrm{~L}$ helium cylinder (Model 49617HE; Leland Ltd., South Plainfield, NJ, USA). In addition to acting as the membrane acceptor phase for analyte transport to the mass spectrometer, helium was also used as a buffer gas by the mass spectrometer. A schematic of the entire apparatus is given in Figure 1. For choosing desired sample flows, two three-way valves (Figure 1, valves A1, A2) were located on either side of the membrane inlet to facilitate convenient switching between aqueous or atmospheric samples (vide infra).

\section{Gaseous Sampling}

Sample was drawn into the MIMS system via two solventcleaned microporous stainless steel inlet filters in series (15 and $5 \mu \mathrm{m}$ ) (Model SS-6 F; Swagelok, Solon, OH, USA), and 1/4in. o.d. Teflon transfer lines (Cole Parmer). The air-sampling inlet was mounted on the roof rack of a sport utility vehicle, approximately $30 \mathrm{~cm}$ above the roofline. Upstream from the MIMS inlet system, gaseous samples flowed through a temperatureregulated chamber housing a toluene- $d_{8}$ permeation tube to allow the continuous on-line infusion of IS (Figure 1). Since the permeation of analytes through the membrane is temperature-dependent, for the experiments described below the MIMS interface, permeation chamber, transfer lines, and inlet filters were independently temperature-regulated to $50^{\circ} \mathrm{C}$. To utilize the system for SVOC analyses (data not shown), the inlet filter and transfer lines could be maintained at temperatures up to $200^{\circ} \mathrm{C}$. The sample flow rate was set to the desired value using a rotameter equipped with a precision, 10-turn needle valve (Model FM4312(4); Advanced Specialty Gas Equipment, Middlesex, NJ, USA) and maintained by a mechanical diaphragm pump (Model B-Series Dia-Vac; Air Dimensions, Deerfield Beach, FL, USA) located after the membrane. A pressure gauge (Model PX209-30V15G5V; Omega, Laval, Canada) located on the sample flow line immediately following the membrane interface was used to monitor pressure changes (e.g., resulting from a clogged inlet filter). A second pressure gauge (Model PX209-030G5V; Omega), located between the diaphragm pump and rotameter, was used to monitor possible changes in sample flow rate. A pressure decrease here would suggest a leak in the sampling system, most notably a failure in the sampling pump diaphragm. Pumps and pressure transducers were controlled and monitored using a custom microcontroller (Arduino UNO, Torino, Italy) and inhouse developed software (Labview 2011; National Instruments, Austin, TX, USA). While underway, the system was powered by a bank of four $6 \mathrm{~V}$ lead acid batteries (2011) (Model T-145 Plus; Trojan Battery Company, Santa Fe Springs, CA, USA) or smaller footprint Li-polymer batteries (2012) (Model 4784 AA; Portable Power Corp, Richmond, CA, USA), using the mass spectrometer's on-board $24 \mathrm{~V}$ voltage regulator to ensure a steady voltage supply to critical components.

Prior to field deployment, the system was calibrated for a variety of target analytes (Table 1). Tandem mass spectrometry scans (MS/MS) were optimized for suitable target analytes, and selected ion monitoring (SIM) scans where used in cases where analytes did not fragment well in the cylindrical ion trap (e.g., reduced sulfur compounds), or for those that were highly fragmented under EI conditions (e.g., pinene). A full scan was also performed to survey for any unexpected analytes (see Table 1). Performing each measurement in an interlaced series resulted in a total scan cycle time of $13.5 \mathrm{~s}$ (averaging 10 replicate scans per measurement). Continuous flow gas standards were produced using certified permeation tubes (KinTek, La Marque, TX, USA) housed in a Dynacalibrator gas dilution apparatus (Model 340; VICI Metronics Inc., Poulsbo, WA, USA), with hydrocarbon scrubbed air used for the dilution gas. At an air sampling flow rate of $1850 \mathrm{~mL} / \mathrm{min}$, the inline toluene- $d_{8}$ permeation tube produced a constant IS concentration of $75 \mathrm{ppb}_{\mathrm{v}}$ throughout calibration. Response factors for each analyte (relative to the IS) were obtained by flowing individual gas standards prepared using the Dynacalibrator through the in-line permeation chamber (Table 1). Detection limits reported for each analyte were based upon three times signal to noise ratio.

The satisfactory performance of the in-line permeation tube chamber and sample heat exchanger was confirmed by 


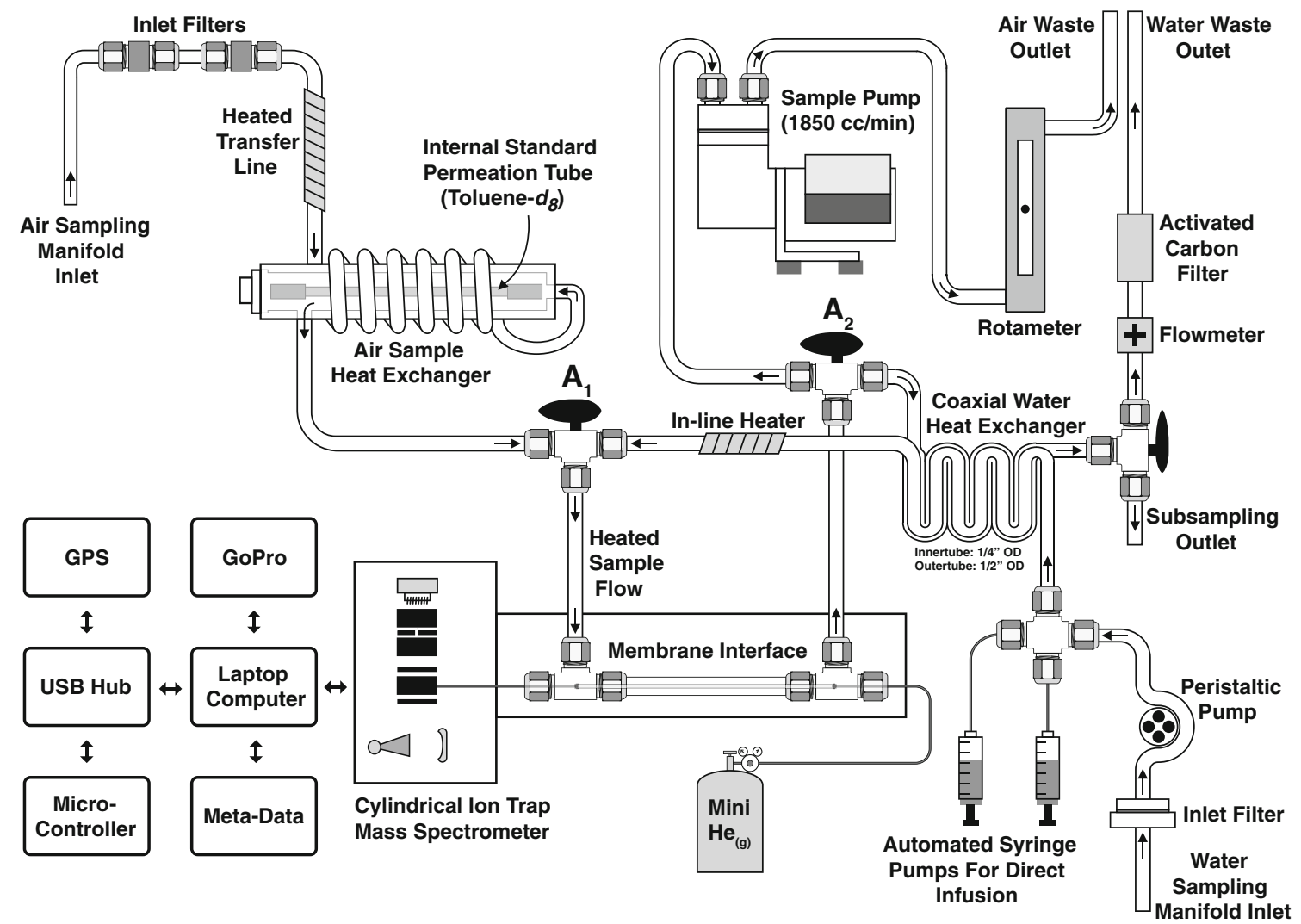

Figure 1. Schematic of the field portable MIMS system. Two flow selection valves (A1 and A2) are used to quickly switch between either the air or aqueous sample introduction manifolds

comparing calibration standards made using the in-line permeation chamber to those made externally using the Dynacalibrator. Figure 2 illustrates the results for gas phase ethylbenzene. Each point on the calibration curve was measured as the mean of 90 scans and fit with a weighted leastsquares solution, using the inverse of the variance as the weighting factor. Importantly, the slopes of each calibration curve are in excellent agreement (within $0.4 \%$ ). This indicates that the in-line permeation chamber and sample heat exchanger perform as anticipated, and field calibrations utilizing the inline chamber for on-line IS addition can be trusted and directly compared with laboratory-based calibrations performed using the Dynacalibrator. A small offset ( $\sim 380$ counts) was observed (Figure 2) as a result of minor instrumental background shifts occurring overnight between the calibration runs, suggesting the necessity of daily background signal checks.

Interference correction factors were also calculated and employed for selected, known isobaric interferents. For example, ethylbenzene EI fragment ions trapped by the MS/MS scan for toluene result in isobaric interference. The fragmentation of ethylbenzene $\left(\mathrm{M}^{+}, m / z\right.$ 106) under EI conditions yields the stable aromatic tropylium ion $\left(\mathrm{C}_{7} \mathrm{H}_{7}^{+}, m / z 91\right)$, which is also used for toluene quantitation via MS/MS $(\mathrm{m} / \mathrm{z} 91 \rightarrow 65)$. This positive isobaric interference of ethylbenzene upon toluene was numerically corrected by using the ratio of isobaric interference between the ethylbenzene $(\mathrm{m} / \mathrm{z} 106 \rightarrow 91)$ and the toluene MS/ MS scans $(m / z \quad 91 \rightarrow 65)$ obtained during the analysis of a gaseous ethylbenzene standard. In this manner, signal contributions from the EI fragment ions of known interferents were numerically corrected. Although a similar, unaccounted for interference from xylene may result in a positive bias for toluene, the low xylene concentrations typically observed (data not shown) will lead to small biases. Future work will focus on resolving additional interference factors using more advanced spectral analysis or soft ionization techniques (e.g., CI) [31].

Experimentally, we have observed that with this system, reproducible measurements are possible for air sampling flow rates of $>1000 \mathrm{~mL} / \mathrm{min}$ (data not shown). In the field, the air sampling flow rate was maintained at $1850 \mathrm{~mL} / \mathrm{min}$ (well above this threshold), yielding a constant $75 \mathrm{ppb}_{\mathrm{v}}$ toluene- $d_{8}$ IS concentration in the sample flow. This resulted in a sufficient IS signal from which analyte concentrations could be calculated using the experimentally determined response factors (Table 1). A sensitivity coefficient was also calculated, giving the user an indication of the overall instrument performance level. For example, if the IS response during an analysis is observed to be $80 \%$ of its response measured during predeployment calibration, a correction factor of 0.8 could be applied to the external calibration coefficients. Drift in the instrumental sensitivity was monitored continuously, allowing for corrective actions (e.g., replace the EI filament, optimize the detector voltage, etc.) when necessary. Thus, continuously infused IS allowed for both real-time calibration and instrument performance monitoring. Variations in operational parameters 


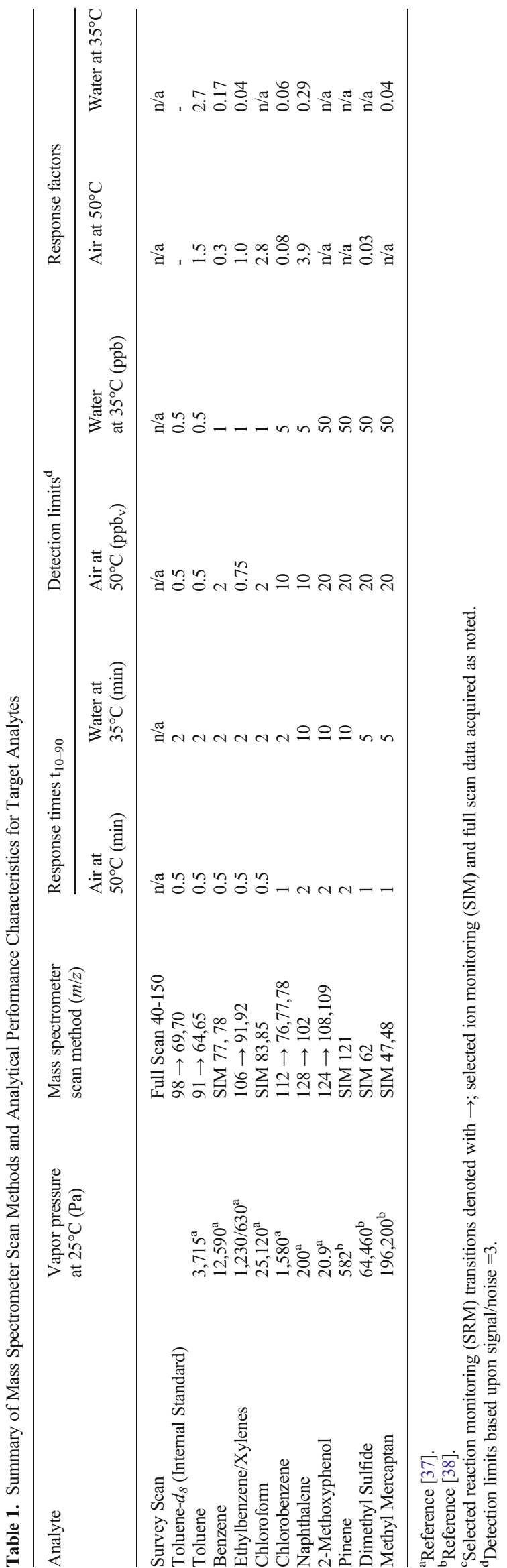

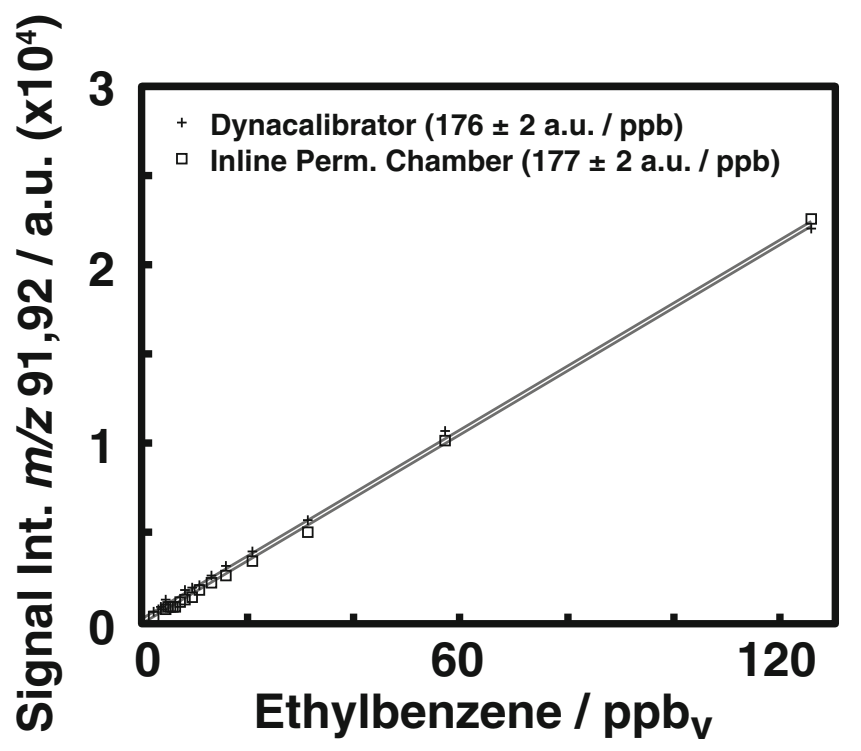

Figure 2. Direct comparison of the instrumental response for gas phase ethylbenzene concentrations with the permeation tube standard located in the Dynacalibrator versus the inline permeation chamber. Instrumental signal intensities are given in arbitrary units (a.u.)

are also detected by observation of the correction coefficient. For example, if the sample temperature, pressure, or flow rate changes, the concentration of the IS will also change, signaling potential problems to the operator during in-field measurements. For this study, we limited our measurements to relatively flat terrain, minimizing pressure variations arising from altitude changes. Sampling parameters were closely monitored and stored as interlaced meta-data to enable post-deployment data interrogation.

Prior to daily deployments during field monitoring campaigns, instrumental background values were reestablished after a $1 \mathrm{~h}$ warm up, using a small cylinder of zero air (Model AI 0.0UM; Praxair, Mississauga, ON, Canada). Periodic calibration checks were performed using toluene and benzene permeation tubes added to the in-line permeation chamber and varying sample gas flow rate from the zero air cylinder.

\section{Aqueous Sampling}

MIMS analysis of aqueous samples was carried out with the same instrumentation by changing the position of valves A1 and A2 (Figure 1). Aqueous sampling was performed at a sample flow rate of $150 \mathrm{~mL} / \mathrm{min}$ using a battery-operated peristaltic pump (Model Masterflex E/S; Cole Parmer, Montreal, Canada) and monitored with a flow meter (Model FLR1000-8; Omega, Laval, Canada). Experimentally, we have observed with this system that reproducible measurements are possible for aqueous sample flow rates of $>100 \mathrm{~mL} / \mathrm{min}$ (data not shown). Incoming aqueous samples were maintained at $35^{\circ} \mathrm{C}$ using a flexible heating tape (thermocouple regulated) wrapped around the sample inlet tubing upstream of the MIMS interface. To reduce the power consumption required for sample heating before measurement, an in-house constructed 
coaxial tubing heat exchanger ( $\sim 70 \%$ efficient) was used to recycle thermal energy from the exiting sample flow (Figure 1). Continuously infused IS was added to the aqueous sample stream, (e.g., toluene- $d_{8}$ ) using a software-controlled syringe pump (Model C24000; Tricontinent, Grass Valley, CA, USA). Additionally, a second syringe pump was incorporated to allow on-line analyte infusions for lab experiments, in-field calibration checks, and standard addition calibrations. The exiting sample flow was passed through an in-house constructed activated carbon filter trap (1000 g of activated carbon suspended between glass wool plugs in a 3 -in. diameter plastic casing) before discharge from the system.

Prior to field deployments, the MIMS system was calibrated for a variety of aqueous analytes using closed 1-L glass recirculation flasks equipped with a Teflon lined septa in a manner similar to that described previously [32, 33]. Deionized water (maintained at $35^{\circ} \mathrm{C}$ by a constant temperature water bath) was recirculated through the MIMS interface at $150 \mathrm{~mL} / \mathrm{min}$, and stepwise injections of combined analyte stock solutions in methanol were used to generate low $\mathrm{ppb}(\mu \mathrm{g} / \mathrm{L})$ aqueous concentrations (e.g., 3 to $30 \mathrm{ppb}$ ) for calibration. To avoid isobaric interferences during aqueous calibrations, two analyte suites were employed: Suite 1: benzene, toluene, chloroform, methyl iodide, dimethyl sulfide; Suite 2: ethylbenzene, chlorobenzene, naphthalene, guaiacol, iso-octane, 1-chloronaphthalene. Toluene- $d_{8}$ was included in both suites and used as an IS for the determination of response factors. Using these data, instrumental background, analyte sensitivity, detection limits, and response factors were determined for each target analyte (Table 1).

For in-field quantitation, incoming aqueous samples were continuously infused with toluene- $d_{8}$ at low ppb levels via a syringe pump, allowing on-line analyte quantitation by response factors as well as affording the continuous monitoring of overall instrument performance. For example, an infusion flow rate of $0.20 \mathrm{~mL} / \mathrm{min}$ of $14 \mathrm{ppm}$ toluene- $d_{8}$ (in methanol) into $150 \mathrm{~mL} / \mathrm{min}$ on-line sample flow rate produced an on-line toluene- $d_{8}$ concentration of $19 \mathrm{ppb}$. The second syringe pump injection port was used to infuse analyte standards in the field for periodic target analyte calibration checks. Some of the aqueous samples were also calibrated by using standard additions methodology, whereupon a calibration solution containing toluene- $d_{8}$ and the desired target analytes was infused into the sample stream immediately following a sample measurement. Field samples included 4-L grab samples collected in amber glass bottles (Scientific Specialties Inc., Hanover, MD, USA) and also the continuous sampling of water directly drawn from rivers and streams over multi-hour sampling periods with the peristaltic pump. Extended periods of stationary aqueous monitoring in the field $(>12 \mathrm{~h})$ were enabled by switching to supplementary power, provided by a small gasoline-powered generator (EU2000i; Honda, Tokyo, Japan) located at least $100 \mathrm{~m}$ downwind from the sampling location. This prevented the complete depletion of the battery systems, and ensured uninterrupted data collection.

\section{Software Development}

Mass spectrometric scan methods were developed and executed using the mass spectrometer's operating software (Griffin System Software, GSS, v3.9; FLIR Systems, West Lafayette, IN, USA). Calibration software to quantitate the raw data and automate the handling of the associated meta-data was written in-house using Labview. Calibration results were stored in a custom file format that was subsequently employed by additional Labview-based software designed for real-time data collection and analysis. Briefly, the real-time software extracted quantitative ion signals from the raw GSS data files and applied the calibration data file, producing real-time quantitative results. These results were then plotted as multiple time series and as color proportional tracks on a map. Maps were downloaded as needed using Google Maps API. All data were automatically stored on Dropbox servers via the cellular data connection. After all mobile data were collected, data workup and re-analysis were performed using in-house developed MATLAB code (Mathworks, Natick, MA, USA). Meta-data and final result files were stored as time series for inspection and quality control. Final results were saved as .kmz files for presentation in Google Earth. These self-contained data files enable inspection of data both geospatially and temporally, are easily networked, and contain hierarchal data structures to enable the presentation and sharing of the large complex datasets in a clear and concise way.

\section{Field Work Methods}

The field monitoring examples presented were obtained in Northern Alberta in early July, 2012. Air sampling data sets were obtained in and around a Steam Assisted Gravity Drainage (SAGD) pilot facility (Statoil Canada Ltd.) located roughly $200 \mathrm{~km}$ south of Fort McMurray, and on public roads north of Fort McMurray along Highway 63. The MIMS system was operated in a sport utility vehicle (GMC Yukon). Stationary air sampling data sets were collected with the vehicle engine off and correlated with meteorological data determined at the sampling site. Aqueous sampling included the analysis of grab samples of on-site processed and ground water as well as measurements made by continuously sampling from nearby natural surface waters.

An example air monitoring dataset, presented here (vide infra), was generated during travel north of Fort McMurray on Highway 63 through areas adjacent to active oil sand recovery operations. Frequent stops (with the vehicle engine off) were performed to allow the collection of meteorological data and to obtain periods of stationary MIMS data. The example dataset was collected on July 7, 2012. The day was warm $\left(27^{\circ} \mathrm{C}\right)$ and dry $(30 \%$ relative humidity), with gentle wind speeds ranging between 4 and $7 \mathrm{~m} / \mathrm{s}$.

Aqueous samples of both on-site process water and off-site natural (stream, river, and ground) water were also collected during this field trip. An example dataset shown here was collected July 3, 2012 from an active steam generation system used by a Statoil Canada Ltd. oil recovery operation (vide infra). The SAGD site has been operational since 2010, and 
the small settling pond is used for holding recovery water prior to reuse in the steam injection process.

\section{Results and Discussion}

\section{Field Work - Air Monitoring}

Data collected with the mass spectrometer operating continuously in a moving vehicle is presented as a time series in Figure 3, and the progression of data workup is presented in each subpanel. The raw data for toluene measurement is shown in Subpanel a and indicates several toluene detections throughout the day. Generally, the data consists of long periods where toluene was not detected, punctuated periodically by transient excursions to higher signal levels. For example, at 16:02, a very strong signal was observed (14,240 counts) when the vehicle passed over a stretch of road that had been very recently paved with fresh asphalt. At 16:24, a sustained signal was detected while the vehicle was stationed downwind of a tailings settling pond used by an active heavy oil extraction operation. Several other minor concentration excursions above detection limit levels may have been the result of passing vehicle exhaust emissions or air masses influenced by other settling ponds. Detections of vehicle exhaust were rather infrequent because of the elevated sampling intake, the very large dilution that rapidly occurs in the turbulence behind a moving vehicle (roughly estimated to be 25,000:1 at highway velocity), and the effective catalytic conversion of hydrocarbon emissions that occurs for hot, underway vehicles [34].

As noted above, ethylbenzene readily fragments under EI to yield $m / z 91$, and this can lead to a positive bias in the toluene concentration as determined by $m / z 91 \rightarrow 65$. To compensate for this, we independently measure ethylbenzene by measuring $\mathrm{m} / \mathrm{z}$ $106 \rightarrow 91$ and applying an interference factor calculated during predeployment calibration. The interference contribution data were smoothed (five-point boxcar) prior to being subtracted from to the analyte signal. Smoothing prevents the inclusion of additional instrumental noise into the signal data and allows signal corrections to be made on a time scale comparable with the MIMS response time for ethylbenzene (Table 1). The contribution of ethylbenzene to the $m / z 91 \rightarrow 65$ signal is typically small (e.g., during the detection of emissions from fresh asphalt, $1.2 \%$,
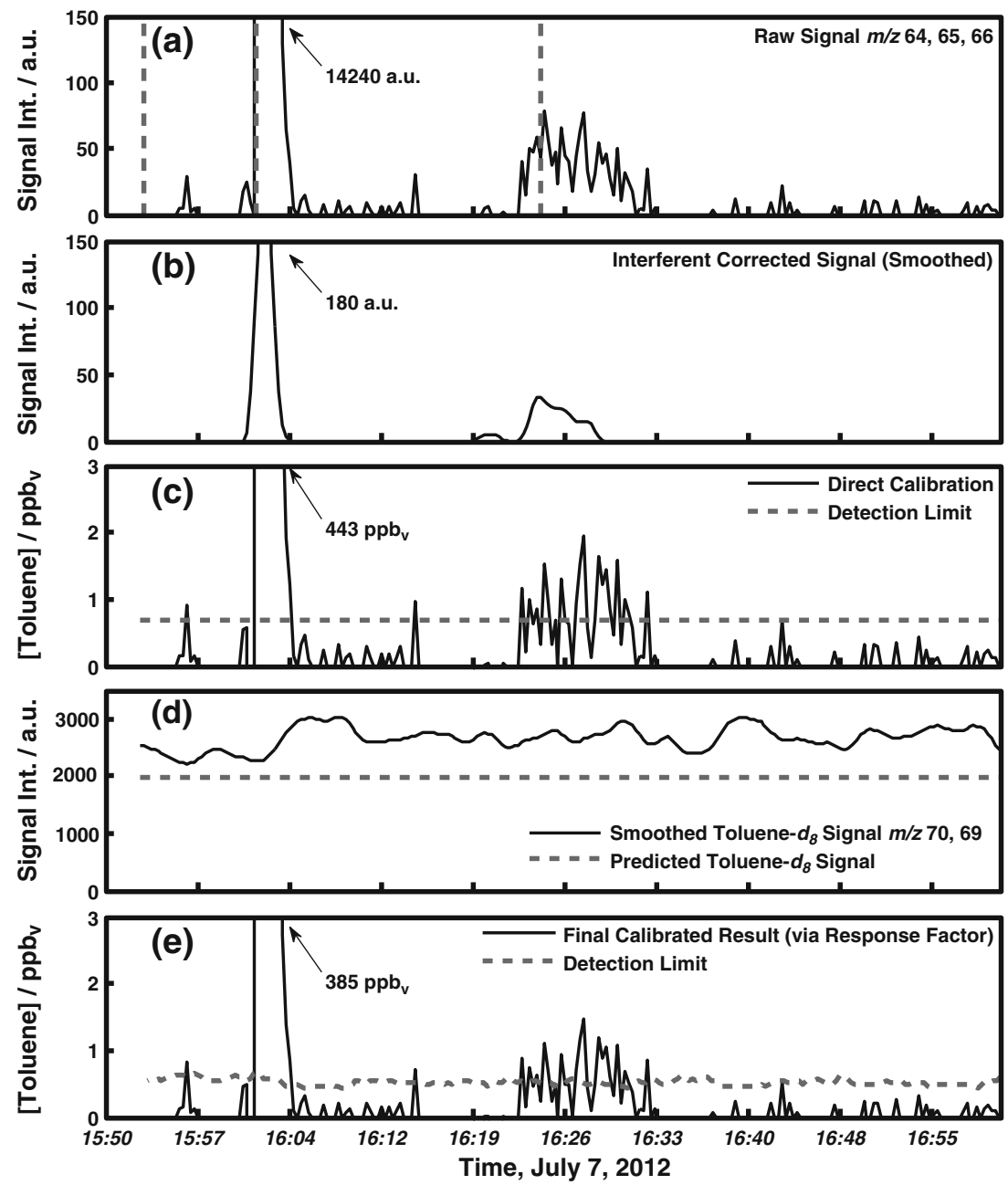

Figure 3. Time series representation of the instrumental data used for real-time toluene quantitation collected north of Fort McMurray, Alberta, Canada. The x-axis represents the time of day when the data was collected. Subpanels show data progression through the data workup process and the final data time series result is given in subpanel $\mathbf{e}$ 
16:02), but can in some instances be significant (e.g., during detection of settling pond off gassing, 40\%, 16:24). The variability, of course, is strongly dependent on the particular sample composition. We cannot rule out positive interference in the toluene quantitation arising from other alkylbenzenes and hydrocarbons likely present near fresh asphalt paving [35]. Therefore, we caution the reader not to over-interpret the displayed toluene concentrations, even after correction for specific interferences such as those arising from ethylbenzene. Although EI is relatively simple to implement, it is a 'hard' ionization strategy, resulting in the extensive fragmentation of many analytes, especially alkyl hydrocarbons. Further improvements in the use of interference factors as well as on-line standard addition strategies and softer ionization sources are currently being investigated to address the challenges of resolving isobaric interferents.

The ethylbenzene corrected toluene signal is converted to concentration in Subpanel c (Figure 3) using direct calibration factors from gaseous calibration standards prepared predeployment. Displaying this data along with the calculated detection limits is an additional quality control check and allows the user to quickly assess any signal deviations in the final data time series (Subpanel e).

Subpanel d (Figure 3) shows the instrumental signal for the continuously infused IS, toluene- $d_{8}(\mathrm{~m} / \mathrm{z} 98 \rightarrow 70)$, and is compared with the expected response to the IS given the sample flow rate and laboratory predeployment calibration (predicted toluene$d_{8}$ signal). The IS concentration was calculated from the analyte constant, $K_{0} \quad$ (cc/mg), derived from molecular weight and the ideal gas constant, permeation tube emission rate, $(E, \mathrm{ng} / \mathrm{min})$, and sampling gas flow rate $(\mathrm{F}, \mathrm{mL} / \mathrm{min}$ at $\mathrm{STP})($ Eq. 1). Expected IS response is calculated as the product of IS concentration $[I S]$ and instrumental sensitivity to the IS $\left(m_{I S}\right)$. The ratio of actual response to expected response provides a sensitivity coefficient that is a real-time measure of the instrument performance (Eq. 2). For example, rapid changes in the sensitivity coefficient may be indicative of transient events that can impact instrument sensitivity (e.g., due to a matrix interference or space-charge effects resulting from a highly contaminated sample or from membrane rupture/fouling). Long-term changes are useful in reporting drift in the instrumental state (e.g., filament or electron multiplier wear). As can be seen in Subpanel d (Figure 3), the instrument was slightly more sensitive to toluene- $d_{8}$ on this deployment than during predeployment lab-based calibrations. The IS data was also boxcar smoothed with a moving average $(n=5)$ to reduce the addition of unnecessary electrical noise to the analyte signals, while allowing sensitivity corrections to be made on a time scale that is comparable to the MIMS signal response times (Table 1).

$$
[I S]=\frac{K_{0} * E}{F}
$$

Sens Coeff $=\frac{\text { Counts }_{I S}}{m_{I S} *[I S]}$

Subpanel e (Figure 3) shows the toluene concentration time series calibrated from an instrumental response factor (e.g., toluene/toluene- $d_{8}$ ) determined during lab calibration, the raw analyte counts and the smoothed IS counts along with the sensitivity adjusted detection limit ( $D L_{\mathrm{Adj}}$, Eq. 3). As the instrumental sensitivity declines during multi-day deployments, the adjusted detection limit will increase. This can happen, for example, when the EI filament is nearing failure. When the detection limit crosses a predetermined threshold, the user can be warned by the software interface and corrective actions (e.g., replace the filament) can be taken. It is important for the instrument field operator to have real-time knowledge of the detection limit for immediate assessment of anomalous signals and to ensure the detection limits are adequate for the collection of meaningful data.

$D L_{A d j}=D L^{*}$ Sens Coeff

As a final data assessment, Subpanel a (Figure 3) indicates two distinct detections. These detections are marked with vertical dashed bars (16:02 and 16:24) and for these intervals, the $30 \mathrm{~s}$ of full scan data following the vertical bar were averaged and presented as insets in Figure 4. The spectra were normalized using the IS signal (measured at $\mathrm{m} / \mathrm{z}$ 98), then corrected by subtracting the spectrum for the IS. The IS spectrum was determined immediately prior to collection of this time series, and this time stamp is also marked with a vertical bar in Subpanel a (Figure 3, 15:54 h). The spectra indicate a complex suite of analytes permeating the membrane. The spectra insets in Figure 4 are distinctly different and suggest that MIMS full scan $m / z$ data can be used in multivariate statistical approaches, such as Principal Component Analysis (PCA) for source apportionment and tracking. They also emphasize the importance of employing selective techniques such as MS/MS for quantitation.

The response factor calibrated toluene mass spectrometric data $(\mathrm{m} / \mathrm{z} 91 \rightarrow 65)$ collected north of Fort McMurray, Canada (Figure 3, Panel e) was time-matched to GPS data and meteorological data, and is presented in Figure 4. White dots represent data collected that were below the detection limit. Wind vectors are represented by the red arrows and can be queried (with a computer mouse click) in Google Earth to inspect the data file source, wind direction, wind speed, temperature, humidity, barometric pressure, time, latitude, and longitude. The 16:02 detection discussed above is noticeable as a large dot just north of Fort McMurray, and the 16:24 detection is labeled at "Settling pond 16:24." The data track presented in Figure 4 is a small subset of a much larger dataset navigable by Google Earth.

Multiple analyte concentrations are embedded in the Google Earth *.kml data file(s) and can be depicted as individual tracks for inspection in real-time or post-deployment. Figure 5 shows a $5 \mathrm{~h}$ continuous time series collected while driving and parked in a discrete pattern (termed a 'Rosette') at strategic locations in and around a SAGD demonstration facility, similar to the methodology employed in other work by our group [17]. Subpanel a illustrates the data obtained for benzene, toluene, and ethylbenzene (+xylene). The shaded regions represent data 


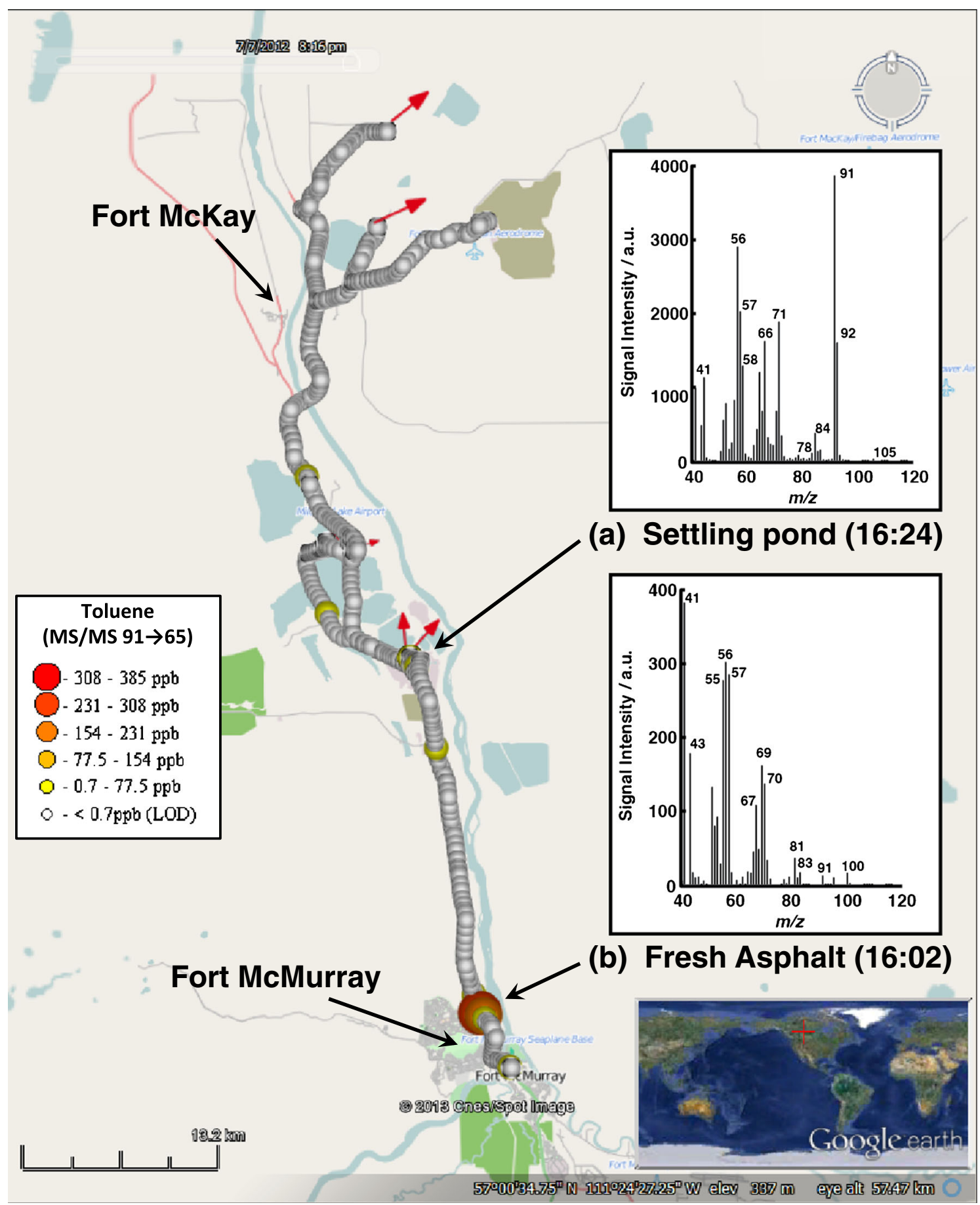

Figure 4. Google Earth snapshot of atmospheric toluene data collected north of Fort McMurray, Alberta, Canada. Data are multidimensional and time-stamped, allowing for a fully navigable data environment and video creation. The insets illustrate full scan mass spectra of selected survey data obtained during detection events. Inset (a) shows the mass spectrum of the air space downwind of a waste-water settling pond (16:24) and (b) shows the mass spectrum for atmospheric monitoring while travelling on a freshly paved asphalt roadway (16:02). Both spectra were normalized to the toluene- $d_{8}$ IS, with background and IS spectra subtracted

collected while the vehicle was stationary (with the vehicle engine off), whereas the unshaded data was collected while moving. Most of the time VOC concentrations are well below our observed detection limits (dashed horizontal line). The signal appearing around 12:00 $\mathrm{h}$ was observed while the vehicle was parked just downwind of a relatively small, lined settling pond used to hold process reuse water. Subpanel b displays a whisker plot of the stationary data only and illustrates the distribution of chemical concentrations as $25-75$ percentiles as well as outliers. Subpanel c illustrates a Google Earth screenshot depicting the toluene concentration track. The navigation panel on the left sidebar of the screen 
(a)
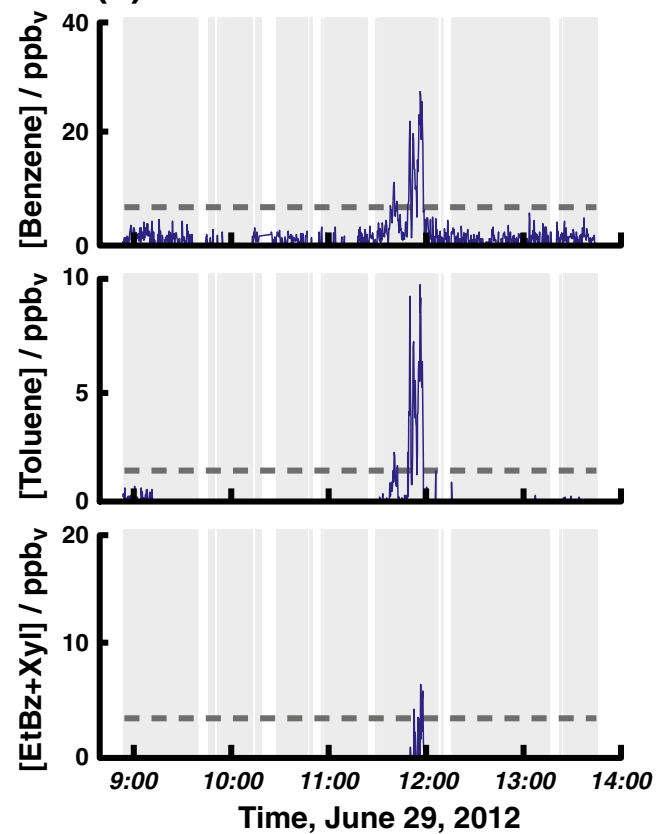

(b)
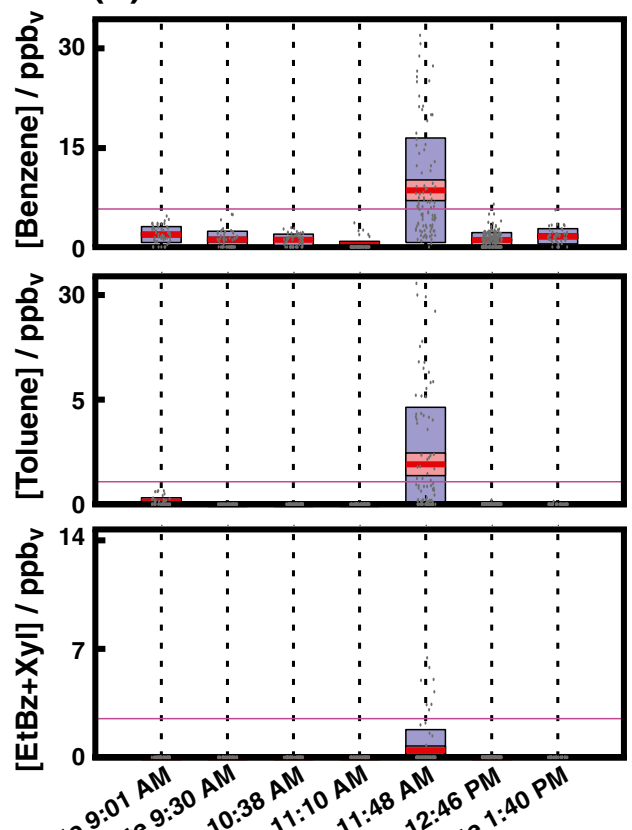

(c)

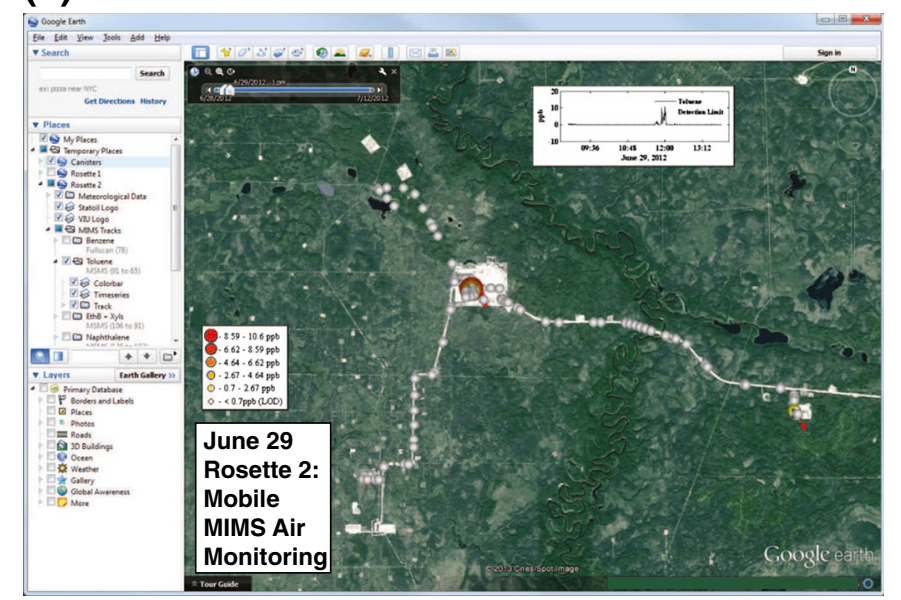

Figure 5. (a) Five-hour time series for atmospheric benzene, toluene, and ethylbenzene + xylenes (EtBz + Xyl) showing data collected in moving vehicle (white background) and while stationary (gray background). The horizontal dotted line represents the calculated detection limits. (b) Whisker box plots for stationary deployments only showing mean concentrations, 25-75 percentiles and data outliers. (c) Google Earth screenshot of the atmospheric toluene data collected on-site. Geospatial time series data for all analytes can be inspected in a single * ${ }^{*} \mathrm{kmz}$ file along with the relevant meta-data

allows the user to select the analyte to display. The data can be viewed in a 'video' mode, playing back the data evolution over time. Screen overlays that include legend, data time series, and institutional logos can also be generated when desired. Importantly, each data point can be queried for its source file, scan information, raw counts, concentration, detection limit, time, latitude, and longitude. In summary, Google Earth has proven to be an extremely useful tool for data visualization and analysis due to its data structure and wide availability.

\section{Field Work - Aqueous Monitoring}

Water samples can be readily analyzed using the same membrane interface and ion trap mass spectrometer system by diverting the aqueous sample stream into the MIMS interface using the aqueous sampling manifold (Figure 1, valves A1 and A2). Continuously infused IS delivered via a syringe pump can be utilized for real-time calibrations using predetermined response factors as above. The other syringe pump (Figure 1) can be loaded with analyte standards and employed for periodic in- 
field calibrations by standard addition methods. The in-line sample heater and heat exchanger system can be used to preheat samples and improve response times, if necessary. Figure 6 illustrates data for the analysis of a VOC contaminated boiler feed water sample, diluted 24:1 to avoid space charge effects in the ion trap from excessively high analyte concentrations. The sample inlet line was switched from deionized water to diluted boiler feed water at 21:24. Data analysis indicated the diluted sample had a concentration of 29 ppb. At 21:39 (dashed bar A), the continuous infusion of a standard solution containing both benzene (19 ppb) and toluene- $d_{8}(19 \mathrm{ppb})$ was initiated. The software system allows for sample analysis by IS and/ or standard addition methods, depending on the injected solution. The benzene signal was calibrated using the standard addition data, whereas the toluene- $d_{8}$ is shown as a demonstration of the use of an IS. Once again, the use of an IS relays important information to the field operator about instrument performance by comparing on-site signal intensities to those observed predeployment. The sudden jump in signal at 21:50 is a result of an entrained air bubble that occurred when switching the sample stream to a washout blank (DI water). The abrupt change observed for the IS signal would be a suitable trigger to suggest that this is compromised data, allowing it to be censored (if desired) from any further data analyses.

Since the instrument can be employed to report continuous, calibrated VOC concentrations in aqueous samples in realtime, it is a powerful in-field monitoring tool for dynamic chemical systems. Industrial sites such as SAGD operations have numerous operational systems to process and reuse water supplies. On-line process monitoring can provide important information to industrial operators. Furthermore, sensitive and selective on-site monitoring of contaminants in surrounding surface and ground waters aids in better environmental stewardship and protection initiatives.

\section{Implications and Future Work}

The combination of instrumentation, data analysis, and visualization software presented here provides a novel approach that

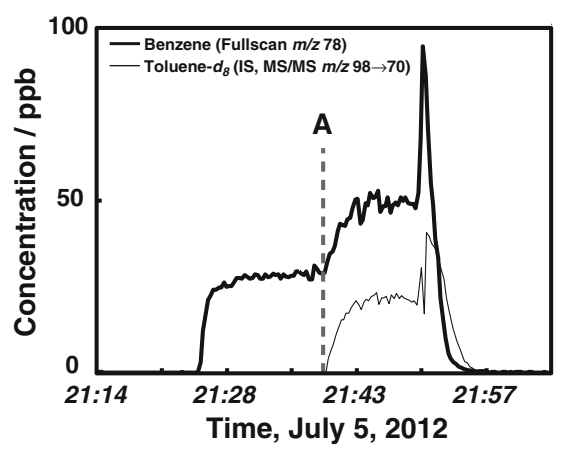

Figure 6. In-field analysis of a heavy oil process water sample. At 21:39 (dashed bar A), an infusion containing both benzene (19 ppb) and toluene- $d_{8}$ IS (19 ppb) commenced. Pre-analysis dilution was 24-fold provides quantitative, information-rich data sets that enable real-time, on-site decision making. This is especially useful for timely process control decisions, emergency response scenarios, adaptive sampling during field measurement campaigns, and long-term environmental monitoring studies. The presented system is amenable to several improvements including the inclusion of a thermally assisted membrane [33] to improve detection limits for SVOC analytes and the implementation of various chemical ionization methods to improve instrument selectivity $[28,30,36]$. Future software development will focus on cataloguing data for improved online database access, additional data input features (e.g., real-time dilution calculations), and improved data (and meta-data) presentation for real-time quality control analysis. Additional peripheral equipment development will include continuous wind vector determinations and simultaneous video monitoring capabilities. The presentation of more extensive datasets including validation studies for hydrocarbons in both air and water will follow in subsequent publications.

\section{Conclusions}

A novel field portable membrane introduction mass spectrometry (MIMS) system is presented that utilizes in-house developed real-time data analysis and visualization software for geospatial mapping of VOC/SVOCs in the environment. The system has been used for monitoring hydrocarbon contaminants in air and water samples during in-field sampling campaigns in and around oil and gas extraction facilities in Northern Alberta, including the direct, in-field measurement of process waters. Real-time detection limits are in the low ppb range. The presented system allows operators to develop intelligent, adaptive sampling strategies based on data being produced in real-time, and provides data to enable remotely located decision makers to implement corrective actions when needed. Continuous mass spectral time series datasets are obtained, processed, and visualized using in-house developed software. Data are temporally resolved and, therefore, capable of capturing transient concentration excursions. In the case of air monitoring, data capture over a wide spatial area by using the system in a moving vehicle is also possible. Although the presented system is currently limited to electron impact ionization (EI), we are exploring the use of softer chemical ionization strategies to alleviate isobaric interferences that arise from the EI fragmentation of matrix species.

\section{Acknowledgments}

The authors gratefully acknowledge Alexander Thompson, Oyvind Mikkelsen, Rudolf Schmid, and the students and staff of the Applied Environmental Research Laboratory (AERL) for their assistance with this project. Special thanks are given to Gregory Vandergrift (AERL) for his assistance with preparing the technical illustrations. The authors thank Vancouver Island University and the University of Victoria for their ongoing 
support of their research and graduate students. This work was supported in part by funding from Statoil ASA (Grant No 4502457485).

\section{References}

1. Liu, Y., Shao, M., Fu, L.L., Lu, S.H., Zeng, L.M., Tang, D.G.: Source profiles of volatile organic compounds (VOCs) measured in China: Part I. Atmos. Environ. 42, 6247-6260 (2008)

2. Simpson, I.J., Blake, N.J., Barletta, B., Diskin, G.S., Fuelberg, H.E., Gorham, K., Huey, L.G., Meinardi, S., Rowland, F.S., Vay, S.A., Weinheimer, A.J., Yang, M., Blake, D.R.: Characterization of trace gases measured over Alberta oil sands mining operations: 76 speciated C-2-C-10 volatile organic compounds (VOCs), $\mathrm{CO} 2, \mathrm{CH} 4, \mathrm{CO}, \mathrm{NO}, \mathrm{NO} 2, \mathrm{NOy}, \mathrm{O}-3$ and SO2. Atmos. Chem. Phys. 10, 11931-11954 (2010)

3. Joint Community Update: Reporting on our Environmental Activities to the Community, Wood Buffalo Environmental Association, Cummulative Environmental Management Association, and Regional Aquatics Monitoring Program, Edmonton, Alberta, Canada (2008)

4. An Assessment of the Environmental Implications of Oil and Gas Production: A Regional Case Study, United States Environmental Protection Agency (2008)

5. McKenzie, L.M., Witter, R.Z., Newman, L.S., Adgate, J.L.: Human health risk assessment of air emissions from development of unconventional natural gas resources. Sci. Total Environ. 424, 79-87 (2012)

6. Chambers, A.K., Strosher, M., Wootton, T., Moncrieff, J., McCready, P.: Direct measurement of fugitive emissions of hydrocarbons from a refinery. J. Air Waste Manage. Assoc. 58, 1047-1056 (2008)

7. Camilli, R., Duryea, A.N.: Characterizing spatial and temporal variability of dissolved gases in aquatic environments with in situ mass spectrometry. Environ. Sci. Technol. 43, 5014-5021 (2009)

8. Kelly, E.N., Short, J.W., Schindler, D.W., Hodson, P.V., Ma, M.S., Kwan, A.K., Fortin, B.L.: Oil sands development contributes polycyclic aromatic compounds to the Athabasca River and its tributaries. Proc. Natl. Acad. Sci. U. S. A. 106, 22346-22351 (2009)

9. Groat, C., Grimshaw, T.: Fact-Based Regulation for Environmental Protection in Shale Gas Development. The Energy Institute, Austin (2012)

10. Lane, D.A.: Mobile mass-spectrometry. Environ. Sci. Technol. 16, A38A46 (1982)

11. Taylor, S., Bierbaum, V.M.: Focus on harsh environment mass spectrometry. J. Am. Soc. Mass Spectrom. 19, 1375-1376 (2008)

12. Herndon, S.C., Jayne, J.T., Zahniser, M.S., Worsnop, D.R., Knighton, B., Alwine, E., Lamb, B.K., Zavala, M., Nelson, D.D., McManus, J.B., Shorter, J.H., Canagaratna, M.R., Onasch, T.B., Kolb, C.E.: Characterization of urban pollutant emission fluxes and ambient concentration distributions using a mobile laboratory with rapid response instrumentation. Faraday Discuss. 130, 327-339 (2005)

13. Guimbaud, C., Catoire, V., Gogo, S., Robert, C., Chartier, M., LaggounDefarge, F., Grossel, A., Alberic, P., Pomathiod, L., Nicoullaud, B., Richard, G.: A portable infrared laser spectrometer for flux measurements of trace gases at the geosphere-atmosphere interface. Meas. Sci. Technol. 22 (2011)

14. Petron, G., Frost, G., Miller, B.R., Hirsch, A.I., Montzka, S.A., Karion, A., Trainer, M., Sweeney, C., Andrews, A.E., Miller, L., Kofler, J., Bar-Ilan, A., Dlugokencky, E.J., Patrick, L., Moore, C.T., Ryerson, T.B., Siso, C., Kolodzey, W., Lang, P.M., Conway, T., Novelli, P., Masarie, K., Hall, B., Guenther, D., Kitzis, D., Miller, J., Welsh, D., Wolfe, D., Neff, W., Tans, P.: Hydrocarbon emissions characterization in the Colorado Front Range: a pilot study. J. Geophys. Res.: Atmos. 117, D04304 (2012)

15. Cooks, R.G., Mueller, T.: Through a glass darkly: glimpses into the future of mass spectrometry. Mass Spectrom. 2, S0001 (2013)

16. Etzkorn, J.M., Davey, N.G., Thompson, A.J., Creba, A.S., Leblanc, C.W., Simpson, C.D., Krogh, E.T., Gill, C.G.: The use of MIMS-MS-MS in field locations as an on-line quantitative environmental monitoring technique for trace contaminants in air and water. J. Chromatogr. Sci. 47, 57-66 (2009)

17. Davey, N.G., Fitzpatrick, C.T.E., Etzkorn, J.M., Martinsen, M., Crampton, R.S., Onstad, G.D., Larson, T.V., Yost, M.G., Krogh, E.T., Gilroy, M., Himes, K.H., Saganic, E.T., Simpson, C.D., Gill, C.G.: Measurement of spatial and temporal variation in volatile hazardous air pollutants in
Tacoma, WA using a mobile membrane introduction mass spectrometry (MIMS) system. J. Environ. Sci. Health A 49, 1199-1208 (2014)

18. Lindinger, W., Hansel, A., Jordan, A.: Proton-transfer reaction mass spectrometry (PTR-MS): on-line monitoring of volatile organic compounds at pptr levels. Chem. Soc. Rev. 27, 347-354 (1998)

19. Chen, H., Xia, Z.N., Pedersen-Bjergaard, S., Svensmark, B., Lauritsen, F.R.: Analysis of semivolatile pharmaceuticals and pollutants in organic micro extracts using hot cell membrane inlet mass spectrometry. Anal. Chem. 81, 4010-4014 (2009)

20. Davey, N.G., Krogh, E.T., Gill, C.G.: Membrane introduction mass spectrometry (MIMS). Trends Anal. Chem. 30, 1477-1485 (2011)

21. Wenner, P.G., Bell, R.J., van Amerom, F.H.W., Toler, S.K., Edkins, J.E., Hall, M.L., Koehn, K., Short, R.T., Byrne, R.H.: Environmental chemical mapping using an underwater mass spectrometer. Trends Anal. Chem. 23, 288-295 (2004)

22. Ouyang, Z., Noll, R.J., Cooks, R.G.: Handheld miniature ion trap mass spectrometers. Anal. Chem. 81, 2421-2425 (2009)

23. Camilli, R., Reddy, C.M., Yoerger, D.R., Van Mooy, B.A.S., Jakuba, M.V., Kinsey, J.C., McIntyre, C.P., Sylva, S.P., Maloney, J.V.: Tracking hydrocarbon plume transport and biodegradation at deepwater horizon. Science 330, 201-204 (2010)

24. Brkic, B., France, N., Taylor, S.: Oil-in-water monitoring using membrane inlet mass spectrometry. Anal. Chem. 83, 6230-6236 (2011)

25. Johnson, R.C., Cooks, R.G., Allen, T.M., Cisper, M.E., Hemberger, P.H.: Membrane introduction mass spectrometry: trends and applications. Mass Spectrom. Rev. 19, 1-37 (2000)

26. Ketola, R.A., Kotiaho, T., Cisper, M.E., Allen, T.M.: Environmental applications of membrane introduction mass spectrometry. J. Mass Spectrom. 37, 457-476 (2002)

27. Overney, F.L., Enke, C.G.: A mathematical study of sample modulation at a membrane inlet mass spectrometer-potential application for the analysis of mixtures. J. Am. Soc. Mass Spectrom. 7, 93-100 (1996)

28. Jordan, A., Haidacher, S., Hanel, G., Hartungen, E., Herbig, J., Mark, L., Schottkowsky, R., Seehauser, H., Sulzer, P., Mark, T.D.: An online ultrahigh sensitivity proton-transfer-reaction mass-spectrometer combined with switchable reagent ion capability (PTR+SRI-MS). Int. J. Mass Spectrom. 286, 32-38 (2009)

29. Prince, B.J., Milligan, D.B., McEwan, M.J.: Application of selected ion flow tube mass spectrometry to real-time atmospheric monitoring. Rapid Commun. Mass Spectrom. 24, 1763-1769 (2010)

30. Smith, D., Spanel, P.: Selected ion flow tube mass spectrometry (SIFT-MS) for on-line trace gas analysis. Mass Spectrom. Rev. 24, 661-700 (2005)

31. Yang, C.H., Khan, N.A., Wu, H.F.: Differentiation and quantification of xylene by combining headspace solid-phase microextraction/gas chromatography and self-ion molecule reaction in an ion trap tandem mass spectrometry. J. Sep. Sci. 31, 3050-3057 (2008)

32. Nelson, J.H., Friesen, D.A., Gill, C.G., Krogh, E.T.: On-line measurement of oxidative degradation kinetics for trace gasoline contaminants in aqueous solutions and natural water by membrane introduction tandem mass spectrometry. J. Environ. Sci. Health A 45, 1720-1731 (2010)

33. Thompson, A.J., Creba, A.S., Ferguson, R.M., Krogh, E.T., Gill, C.G.: A coaxially heated membrane introduction mass spectrometry interface for the rapid and sensitive on-line measurement of volatile and semivolatile organic contaminants in air and water at parts-per-trillion levels. Rapid Commun. Mass Spectrom. 20, 2000-2008 (2006)

34. Kaspar, J., Fornasiero, P., Hickey, N.: Automotive catalytic converters: current status and some perspectives. Catal. Today 77, 419-449 (2003)

35. Gasthauer, E., Maze, M., Marchand, J.P., Amouroux, J.: Characterization of asphalt fume composition by GC/MS and effect of temperature. Fuel $\mathbf{8 7}$, $1428-1434$ (2008)

36. Huang, G.M., Gao, L., Duncan, J., Harper, J.D., Sanders, N.L., Ouyang, Z., Cooks, R.G.: Direct detection of benzene, toluene, and ethylbenzene at trace levels in ambient air by atmospheric pressure chemical ionization using a handheld mass spectrometer. J. Am. Soc. Mass Spectrom. 21, 132135 (2010)

37. Schwarzenbach, R.P., Gschwend, P.M., Imboden, D.M.: Environmental Organic Chemistry. John Wiley and Sons Ltd., Hoboken, NJ, USA (2003)

38. National Institute of Standards and Technology. NIST Chem Webbook. Available at: http://webbook.nist.gov/chemistry/ (2012). Accessed 3 Oct 2014 